JOURNAL OF SYNCHROTRON RADIATION

ISSN 1600-5775

Keywords: PhotonDiag2018 workshop; freeelectron lasers; photon beam diagnostics.

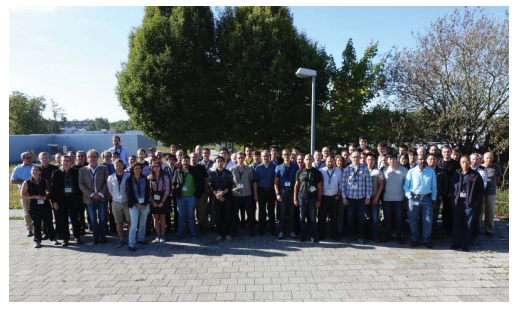

(C) 2020 International Union of Crystallography

\section{Foreword to the special virtual issue dedicated to the proceedings of the PhotonDiag2018 workshop on FEL Photon Diagnostics, Instrumentation, and Beamlines Design}

\author{
Daniele Cocco, ${ }^{\mathrm{a}, \mathrm{b}}$ Jan Grünert, ${ }^{\mathrm{c}}$ Elke Ploenjes, ${ }^{\mathrm{d} *}$ Kai Tiedtke ${ }^{\mathrm{d}}$ and \\ Marco Zangrando ${ }^{\mathbf{e}}$
}

a'Lawrence Berkeley National Laboratory, Berkeley, CA 94720, USA, b'SAC National Accelerator Laboratory, 2575 Sand Hill Road, Menlo Park, CA 94025, USA, 'European XFEL, Holzkoppel 4, 22869 Schenefeld, Germany, ${ }^{\mathbf{d} D e u t s c h e s}$ Elektronen-Synchrotron, Notkestraße 85, 22603 Hamburg, Germany, and ${ }^{\mathbf{e}}$ Elettra - Sincrotrone Trieste SCpA and IOM-CNR, SS 14 km 163.5, Trieste, Italy. *Correspondence e-mail: elke.ploenjes@desy.de

From the pioneering early days of synchrotron radiation, with rings like Tantalus, SURF, ADONE and DESY among others, to the third-generation storage rings, the quest for better photon transport systems has been stimulated by inventive users and by competition for users and funding. This quest drove major breakthroughs in optics, coating and optical design (McKinney \& Howells, 1980; Padmore, 1989; Johnson, 1986; Howells, 1980; Harada \& Kita, 1980; Reininger \& Saile, 1990; Chen \& Sette, 1989; Petersen, 1982; Reininger \& de Castro, 2005; Cocco et al., 1999; Follath, 2001; Naletto \& Tondello, 1992), metrology (von Bieren, 1982; Takacs \& Qian, 1989; Irick \& McKinney, 1997; Cocco et al., 2003; Siewert et al., 2004; Vivo \& Barrett, 2017) and simulation codes (Lai \& Cerrina, 1986; Raimondi \& Spiga, 2015; Sanchez del Rio \& Rebuffi, 2019; Rebuffi \& Sanchez del Rio, 2017; Shi et al., 2014). New optical designs have been proposed to meet the request for new experiments, challenging the optical manufacturers to improve their polishing capabilities or coating quality and the metrologists to improve their ability in measuring the optics. The result has been that better beamlines have been put into operation and more sophisticated experiments have been performed. This virtuous loop generated a constant and steady progress in all the photon transport system related instrumentation and techniques. However, a novel approach to optics and optical design, and, somehow, a revolutionary way of thinking, did not happen until a new kind of facility - the freeelectron laser (FEL) - became operational. Pioneering work carried out at FLASH (the first VUV FEL) (Ackermann et al., 2007; Ayvazyan et al., 2006; Tiedtke et al., 2009), LCLS (the first X-ray FEL) (Emma et al., 2010; White et al., 2015) and FERMI (the first seeded FEL) (Allaria et al., 2010, 2012) paved the way to a new boost in optics development. The development became a clear priority for all facilities with the advent of the European XFEL (Abela et al., 2006; Tschentscher et al., 2017), SACLA (Ishikawa et al., 2012), SwissFEL (Ko et al., 2017) and PAL-XFEL (Milne et al., 2017). New challenges came along, related to the high peak photon pulse energy, short pulse duration, and increasing repetition rates. In the meantime, thanks to the development made at Osaka University (Yamauchi et al., 2002), diffraction-limited optics became available. Thanks to these mirrors, diffraction-limited nano-scale spots, or almost perfect beam out of focus, were made possible (Matsuyama et al., 2016; Cocco et al., 2018). New sources, not only FELs but also the diffraction-limited storage rings (DLSRs) under construction all around the world, will strongly benefit from the work done so far.

A uniquely distinguishing feature that highly differentiates the photon transport system of a FEL from that of a synchrotron radiation source is the need for completely new photon diagnostics (Tiedtke et al., 2009; Moeller et al., 2011; Grünert, 2012), with the ability and necessity to measure the properties of the light pulses with high precision, sometimes even shot by shot. Intensity, for sure, but also spectrum, wavefront, polarization, beam position and pointing and temporal jitter among others are often required to be continuously monitored, ideally in a non-invasive way, to provide real-time information to the experimentalists and to the operators for machine performance optimization. 
To face these challenges, in 2010, under the auspices of the EuroFEL consortium, a first workshop was held in Hamburg, Germany ${ }^{\mathbf{1}}$. It was focused on optimized concepts for the photon beam transport of femtosecond laserlike radiation of short wavelength, including the metrology of optical components and centered on advanced diagnostics for the determination of photon beam properties. Following the first workshop, with the increasing demand in highly performing diagnostics and better optical systems, a second workshop was held in Trieste, Italy, hosted by FERMI in $2015^{2}$ and a third, hosted by the Linac Coherent Light Source at SLAC, in $2017^{3}$. The fourth workshop of this successful

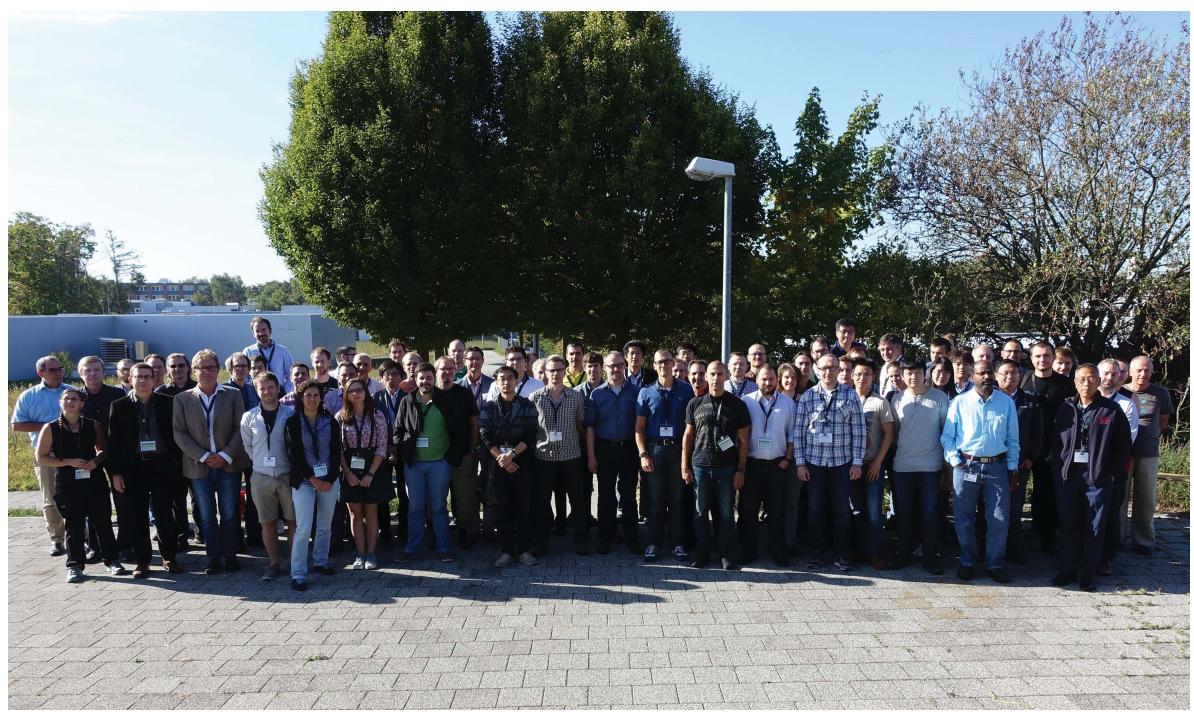

Group photo from the PhotonDiag2018 workshop. series, presented in this proceedings volume, took place, as the original one, in Hamburg in September 2018, co-hosted by DESY, the site of FLASH, and the European XFEL facility. The workshop has been organized under the auspices of the FELs of Europe consortium and has seen the participation of almost 100 scientists and engineers from all over the world (see Fig. 1).

The participants had the chance to attend 40 technical talks, including 7 invited presentations, and 28 posters. The oral presentations spanned from the status and update of the existing (in operation or in advanced state of construction) FEL facilities, to some specific scientific applications of the FEL radiation. But, of course, the central topics covered during the workshop were related to advances in X-ray optics and diagnostics. Among the various novel diagnostic concepts presented, the wavefront sensors played a central role in the workshop, as they became central to the operation of most advanced facilities worldwide. Similarly to the workshop presentations, the 17 articles that are part of these proceedings are describing innovative X-ray optics and novel or improved diagnostics. This is the first virtual issue published in the Journal of Synchrotron Radiation and is intended to give the largest possible access to the researchers and engineers around the world to one of the most dynamically evolving topics in the X-ray science world, e.g. the development of better, ideally non-invasive and shot to shot, photon diagnostics.

This virtual proceeding (https://journals.iucr.org/special_ issues/2020/photondiag2018/index.html) starts with the articles focused on diagnostics to study the spectral distribution, the wavefront, the intensity, the profile, the polarization and the temporal distribution. The combined use of photon diagnostics and electron diagnostics to study machine performance follows after the initial section. The third part deals with more

\footnotetext{
1 https://photondiag2010.eurofel.eu.

2 http://www.elettra.trieste.it/events/2015/PhotonDiag/Main.HomePage.html.

${ }^{3}$ https://portal.slac.stanford.edu/sites/conf_public/photondiag2017.
}

conventional X-ray optics, from metrology over adaptive focusing mirrors to crystal monochromators. The proceedings close with one crucial component to most experiments and diagnostics, e.g. the X-ray detectors.

We hope these high-level articles will seed in you, the reader, new ideas to further advance the demanding field of optics and diagnostics for diffraction-limited sources and inspire you to attend future events of this workshop series and be part of this community.

\section{References}

Abela, R., et al. (2006). XFEL: The European X-ray Free-Electron Laser, Technical Design Report 06-097. DESY, Hamburg, Germany.

Ackermann, W., Asova, G., Ayvazyan, V., Azima, A., Baboi, N., Bähr, J., Balandin, V., Beutner, B., Brandt, A., Bolzmann, A., Brinkmann, R., Brovko, O. I., Castellano, M., Castro, P., Catani, L., Chiadroni, E., Choroba, S., Cianchi, A., Costello, J. T., Cubaynes, D., Dardis, J., Decking, W., Delsim-Hashemi, H., Delserieys, A., Di Pirro, G., Dohlus, M., Düsterer, S., Eckhardt, A., Edwards, H. T., Faatz, B., Feldhaus, J., Flöttmann, K., Frisch, J., Fröhlich, L., Garvey, T., Gensch, U., Gerth, Ch., Görler, M., Golubeva, N., Grabosch, H., Grecki, M., Grimm, O., Hacker, K., Hahn, U., Han, J. H., Honkavaara, K., Hott, T., Hüning, M., Ivanisenko, Y., Jaeschke, E., Jalmuzna, W., Jezynski, T., Kammering, R., Katalev, V., Kavanagh, K., Kennedy, E. T., Khodyachykh, S., Klose, K., Kocharyan, V., Körfer, M., Kollewe, M., Koprek, W., Korepanov, S., Kostin, D., Krassilnikov, M., Kube, G., Kuhlmann, M., Lewis, C. L. S., Lilje, L., Limberg, T., Lipka, D., Löhl, F., Luna, H., Luong, M., Martins, M., Meyer, M., Michelato, P., Miltchev, V., Möller, W. D., Monaco, L., Müller, W. F. O., Napieralski, O., Napoly, O., Nicolosi, P., Nölle, D., Nuñez, T., Oppelt, A., Pagani, C., Paparella, R., Pchalek, N., Pedregosa-Gutierrez, J., Petersen, B., Petrosyan, B., Petrosyan, G., Petrosyan, L., Pflüger, J., Plönjes, E., Poletto, L., Pozniak, K., Prat, E., Proch, D., Pucyk, P., Radcliffe, P., Redlin, H., Rehlich, K., Richter, M., Roehrs, M., Roensch, J., Romaniuk, R., Ross, M., Rossbach, J., Rybnikov, V., Sachwitz, M., Saldin, E. L., Sandner, W., Schlarb, H., Schmidt, B., Schmitz, M., Schmüser, P., Schneider, J. R., Schneidmiller, E. A., Schnepp, S., Schreiber, S., 
Seidel, M., Sertore, D., Shabunov, A. V., Simon, C., Simrock, S., Sombrowski, E., Sorokin, A. A., Spanknebel, P., Spesyvtsev, R., Staykov, L., Steffen, B., Stephan, F., Stulle, F., Thom, H., Tiedtke, K., Tischer, M., Toleikis, S., Treusch, R., Trines, D., Tsakov, I., Vogel, E., Weiland, T., Weise, H., Wellhöfer, M., Wendt, M., Will, I., Winter, A., Wittenburg, K., Wurth, W., Yeates, P., Yurkov, M. V., Zagorodnov, I. \& Zapfe, K. (2007). Nat. Photon. 1, 336-342.

Allaria, E., Appio, R., Badano, L., Barletta, W. A., Bassanese, S., Biedron, S. G., Borga, A., Busetto, E., Castronovo, D., Cinquegrana, P., Cleva, S., Cocco, D., Cornacchia, M., Craievich, P., Cudin, I., D’Auria, G., Dal Forno, M., Danailov, M. B., De Monte, R., De Ninno, G., Delgiusto, P., Demidovich, A., Di Mitri, S., Diviacco, B., Fabris, A., Fabris, R., Fawley, W., Ferianis, M., Ferrari, E., Ferry, S., Froehlich, L., Furlan, P., Gaio, G., Gelmetti, F., Giannessi, L., Giannini, M., Gobessi, R., Ivanov, R., Karantzoulis, E., Lonza, M., Lutman, A., Mahieu, B., Milloch, M., Milton, S. V., Musardo, M., Nikolov, I., Noe, S., Parmigiani, F., Penco, G., Petronio, M., Pivetta, L., Predonzani, M., Rossi, F., Rumiz, L., Salom, A., Scafuri, C., Serpico, C., Sigalotti, P., Spampinati, S., Spezzani, C., Svandrlik, M., Svetina, C., Tazzari, S., Trovo, M., Umer, R., Vascotto, A., Veronese, M., Visintini, R., Zaccaria, M., Zangrando, D. \& Zangrando, M. (2012). Nat. Photon. 6, 699-704.

Allaria, E., Callegari, C., Cocco, D., Fawley, W. M., Kiskinova, M., Masciovecchio, C. \& Parmigiani, F. (2010). New J. Phys. 12, 075002.

Ayvazyan, V., Baboi, N., Bähr, J., Balandin, V., Beutner, B., Brandt, A., Bohnet, I., Bolzmann, A., Brinkmann, R., Brovko, O. I., Carneiro, J. P., Casalbuoni, S., Castellano, M., Castro, P., Catani, L., Chiadroni, E., Choroba, S., Cianchi, A., Delsim-Hashemi, H., Di Pirro, G., Dohlus, M., Düsterer, S., Edwards, H. T., Faatz, B., Fateev, A. A., Feldhaus, J., Flöttmann, K., Frisch, J., Fröhlich, L., Garvey, T., Gensch, U., Golubeva, N., Grabosch, H., Grigoryan, B., Grimm, O., Hahn, U., Han, J. H., Hartrott, M. V., Honkavaara, K., Hüning, M., Ischebeck, R., Jaeschke, E., Jablonka, M., Kammering, R., Katalev, V., Keitel, B., Khodyachykh, S., Kim, Y., Kocharyan, V., Körfer, M., Kollewe, M., Kostin, D., Krämer, D., Krassilnikov, M., Kube, G., Lilje, L., Limberg, T., Lipka, D., Löhl, F., Luong, M., Magne, C., Menzel, J., Michelato, P., Miltchev, V., Minty, M., Möller, W. D., Monaco, L., Müller, W., Nagl, M., Napoly, O., Nicolosi, P., Nölle, D., Nuñez, T., Oppelt, A., Pagani, C., Paparella, R., Petersen, B., Petrosyan, B., Pflüger, J., Piot, P., Plönjes, E., Poletto, L., Proch, D., Pugachov, D., Rehlich, K., Richter, D., Riemann, S., Ross, M., Rossbach, J., Sachwitz, M., Saldin, E. L., Sandner, W., Schlarb, H., Schmidt, B., Schmitz, M., Schmüser, P., Schneider, J. R., Schneidmiller, E. A., Schreiber, H., Schreiber, S., Shabunov, A. V., Sertore, D., Setzer, S., Simrock, S., Sombrowski, E., Staykov, L., Steffen, B., Stephan, F., Stulle, F., Sytchev, K. P., Thom, H., Tiedtke, K., Tischer, M., Treusch, R., Trines, D., Tsakov, I., Vardanyan, A., Wanzenberg, R., Weiland, T., Weise, H., Wendt, M., Will, I., Winter, A., Wittenburg, K., Yurkov, M. V., Zagorodnov, I., Zambolin, P. \& Zapfe, K. (2006). Eur. Phys. J. D, 37, 297-303.

Bieren, K. von (1982). Proc. SPIE, 0343, 101-108.

Chen, C. T. \& Sette, F. (1989). Rev. Sci. Instrum. 60, 1616-1621.

Cocco, D., Marsi, M., Kiskinova, M., Prince, K. C., Schmidt, T., Heun, S. \& Bauer, E. (1999). Proc. SPIE, 3767, 271-279.

Cocco, D., Idir, M., Morton, D., Raimondi, L. \& Zangrando, M. (2018). Nucl. Instrum. Methods Phys. Res. A, 907, 105-115.

Cocco, D., Sostero, G. \& Zangrando, M. (2003). Rev. Sci. Instrum. 74, 3544-3548.

Emma, P., Akre, R., Arthur, J., Bionta, R., Bostedt, C., Bozek, J., Brachmann, A., Bucksbaum, P., Coffee, R., Decker, F., Ding, Y., Dowell, D., Edstrom, S., Fisher, A., Frisch, J., Gilevich, S., Hastings, J., Hays, G., Hering, P., Huang, Z., Iverson, R., Loos, H., Messerschmidt, M., Miahnahri, A., Moeller, S., Nuhn, H., Pile, G., Ratner, D., Rzepiela, J., Schultz, D., Smith, T., Stefan, P., Tompkins, H., Turner, J., Welch, J., White, W., Wu, J., Yocky, G. \& Galayda, J. (2010). Nat. Photon. 4, 641-647.
Follath, R. (2001). Nucl. Instrum. Methods Phys. Res. A, 467-468, 418-425.

Grünert, J. (2012). Conceptual Design Report: Framework for X-ray Photon Diagnostics at the European XFEL, XFEL. EU Technical Report TR-2012-003. European XFEL, Hamburg, Germany.

Harada, T. \& Kita, T. (1980). Appl. Opt. 19, 3987-3993.

Howells, M. R. (1980). Nucl. Instrum. Methods, 177, 127-139.

Irick, S. C. \& McKinney, W. R. (1997). AIP Conf. Proc. pp. 118-123.

Ishikawa, T., Aoyagi, H., Asaka, T., Asano, Y., Azumi, N., Bizen, T., Ego, H., Fukami, K., Fukui, T., Furukawa, Y., Goto, S., Hanaki, H., Hara, T., Hasegawa, T., Hatsui, T., Higashiya, A., Hirono, T., Hosoda, N., Ishii, M., Inagaki, T., Inubushi, Y., Itoga, T., Joti, Y., Kago, M., Kameshima, T., Kimura, H., Kirihara, Y., Kiyomichi, A., Kobayashi, T., Kondo, C., Kudo, T., Maesaka, H., Maréchal, X. M., Masuda, T., Matsubara, S., Matsumoto, T., Matsushita, T., Matsui, S., Nagasono, M., Nariyama, N., Ohashi, H., Ohata, T., Ohshima, T., Ono, S., Otake, Y., Saji, C., Sakurai, T., Sato, T., Sawada, K., Seike, T., Shirasawa, K., Sugimoto, T., Suzuki, S., Takahashi, S., Takebe, H., Takeshita, K., Tamasaku, K., Tanaka, H., Tanaka, R., Tanaka, T., Togashi, T., Togawa, K., Tokuhisa, A., Tomizawa, H., Tono, K., Wu, S., Yabashi, M., Yamaga, M., Yamashita, A., Yanagida, K., Zhang, C., Shintake, T., Kitamura, H. \& Kumagai, N. (2012). Nat. Photon. 6, 540-544.

Johnson, R. L. (1986). Nucl. Instrum. Methods Phys. Res. A, 246, $303-$ 309.

Ko, I., Kang, H., Heo, H., Kim, C., Kim, G., Min, C., Yang, H., Baek, S., Choi, H., Mun, G., Park, B., Suh, Y., Shin, D., Hu, J., Hong, J., Jung, S., Kim, S., Kim, K., Na, D., Park, S., Park, Y., Jung, Y., Jeong, S., Lee, H., Lee, S., Lee, S., Oh, B., Suh, H., Han, J., Kim, M., Jung, N., Kim, Y., Lee, M., Lee, B., Sung, C., Mok, I., Yang, J., Parc, Y., Lee, W., Lee, C., Shin, H., Kim, J., Kim, Y., Lee, J., Park, S., Kim, J., Park, J., Eom, I., Rah, S., Kim, S., Nam, K. H., Park, J., Park, J., Kim, S., Kwon, S., An, R., Park, S., Kim, K., Hyun, H., Kim, S., Kim, S., Yu, C., Kim, B., Kang, T., Kim, K., Kim, S., Lee, H., Lee, H., Park, K., Koo, T., Kim, D. \& Lee, K. (2017). Appl. Sci. 7, 479.

Lai, B. \& Cerrina, F. (1986). Nucl. Instrum. Methods Phys. Res. A, 246, 337-341.

Matsuyama, S., Nakamori, H., Goto, T., Kimura, T., Khakurel, K. P., Kohmura, Y., Sano, Y., Yabashi, M., Ishikawa, T., Nishino, Y. \& Yamauchi, K. (2016). Sci. Rep. 6, 24801.

McKinney, W. R. \& Howells, M. R. (1980). Nucl. Instrum. Methods, 172, 149-156.

Milne, C., Schietinger, T., Aiba, M., Alarcon, A., Alex, J., Anghel, A., Arsov, V., Beard, C., Beaud, P., Bettoni, S., Bopp, M., Brands, H., Brönnimann, M., Brunnenkant, I., Calvi, M., Citterio, A., Craievich, P., Csatari Divall, M., Dällenbach, M., D'Amico, M., Dax, A., Deng, Y., Dietrich, A., Dinapoli, R., Divall, E., Dordevic, S., Ebner, S., Erny, C., Fitze, H., Flechsig, U., Follath, R., Frei, F., Gärtner, F., Ganter, R., Garvey, T., Geng, Z., Gorgisyan, I., Gough, C., Hauff, A., Hauri, C., Hiller, N., Humar, T., Hunziker, S., Ingold, G., Ischebeck, R., Janousch, M., Juranić, P., Jurcevic, M., Kaiser, M., Kalantari, B., Kalt, R., Keil, B., Kittel, C., Knopp, G., Koprek, W., Lemke, H., Lippuner, T., Llorente Sancho, D., Löhl, F., LopezCuenca, C., Märki, F., Marcellini, F., Marinkovic, G., Martiel, I., Menzel, R., Mozzanica, A., Nass, K., Orlandi, G., Ozkan Loch, C., Panepucci, E., Paraliev, M., Patterson, B., Pedrini, B., Pedrozzi, M., Pollet, P., Pradervand, C., Prat, E., Radi, P., Raguin, J., Redford, S., Rehanek, J., Réhault, J., Reiche, S., Ringele, M., Rittmann, J., Rivkin, L., Romann, A., Ruat, M., Ruder, C., Sala, L., Schebacher, L., Schilcher, T., Schlott, V., Schmidt, T., Schmitt, B., Shi, X., Stadler, M., Stingelin, L., Sturzenegger, W., Szlachetko, J., Thattil, D., Treyer, D., Trisorio, A., Tron, W., Vetter, S., Vicario, C., Voulot, D., Wang, M., Zamofing, T., Zellweger, C., Zennaro, R., Zimoch, E., Abela, R., Patthey, L. \& Braun, H. (2017). Appl. Sci. 7, 720.

Moeller, S., Arthur, J., Brachmann, A., Coffee, R., Decker, F., Ding, Y., Dowell, D., Edstrom, S., Emma, P., Feng, Y., Fisher, A., Frisch, J., Galayda, J., Gilevich, S., Hastings, J., Hays, G., Hering, P., Huang, 
Z., Iverson, R., Krzywinski, J., Lewis, S., Loos, H., Messerschmidt, M., Miahnahri, A., Nuhn, H., Ratner, D., Rzepiela, J., Schultz, D., Smith, T., Stefan, P., Tompkins, H., Turner, J., Welch, J., White, B., Wu, J., Yocky, G., Bionta, R., Ables, E., Abraham, B., Gardener, C., Fong, K., Friedrich, S., Hau-Riege, S., Kishiyama, K., McCarville, T., McMahon, D., McKernan, M., Ott, L., Pivovaroff, M., Robinson, J., Ryutov, D., Shen, S., Soufli, R. \& Pile, G. (2011). Nucl. Instrum. Methods Phys. Res. A, 635, S6-S11.

Naletto, G. \& Tondello, G. (1992). Pure Appl. Opt. 1, 347-358.

Padmore, H. A. (1989). Rev. Sci. Instrum. 60, 1608-1615.

Petersen, H. (1982). Opt. Commun. 40, 402-406.

Raimondi, L. \& Spiga, D. (2015). Astron. Astrophys. 573, A22.

Rebuffi, L. \& Sanchez del Rio, M. (2017). Proc. SPIE, 10388, 103880 S.

Reininger, R. \& de Castro, A. R. B. (2005). Nucl. Instrum. Methods Phys. Res. A, 538, 760-770.

Reininger, R. \& Saile, V. (1990). Nucl. Instrum. Methods Phys. Res. A, 288, 343-348.

Rio, M. S. \& Rebuffi, L. (2019). AIP Conf. Proc. 2054, 060081.

Shi, X., Reininger, R., Sanchez del Rio, M. \& Assoufid, L. (2014). J. Synchrotron Rad. 21, 669-678.
Siewert, F., Noll, T., Schlegel, T., Zeschke, T. \& Lammert, H. (2004). AIP Conf. Proc. 705, 847-850.

Takacs, P. Z. \& Qian, S. N. (1989). US Patent U4884697.

Tiedtke, K., Azima, A., von Bargen, N., Bittner, L., Bonfigt, S., Düsterer, S., Faatz, B., Frühling, U., Gensch, M., Gerth, C., Guerassimova, N., Hahn, U., Hans, T., Hesse, M., Honkavaar, K., Jastrow, U., Juranic, P., Kapitzki, S., Keitel, B., Kracht, T., Kuhlmann, M., Li, W. B., Martins, M., Núñez, T., Plönjes, E., Redlin, H., Saldin, E. L., Schneidmiller, E. A., Schneider, J. R., Schreiber, S., Stojanovic, N., Tavella, F., Toleikis, S., Treusch, R., Weigelt, H., Wellhöfer, M., Wabnitz, H., Yurkov, M. V. \& Feldhaus, J. (2009). New J. Phys. 11, 023029.

Tschentscher, T., Bressler, C., Grünert, J., Madsen, A., Mancuso, A., Meyer, M., Scherz, A., Sinn, H. \& Zastrau, U. (2017). Appl. Sci. 7, 592.

Vivo, A. \& Barrett, R. (2017). Proc. SPIE, 10385, 103850N.

White, W. E., Robert, A. \& Dunne, M. (2015). J. Synchrotron Rad. 22, 472-476.

Yamauchi, K., Mimura, H., Inagaki, K. \& Mori, Y. (2002). Rev. Sci. Instrum. 73, 4028-4033. 